\title{
Reinforcers in Infancy: Classical Conditioning Using Stroking or Intra-oral Infusions of Milk as UCS
}

\author{
REGINA M. SULLIVAN \\ W. G. HALL \\ Department of Psychology \\ Duke University \\ Durham, North Carolina
}

Six-day-old rats received 20 forward pairings of an odor-conditioned stimulus (CS) with one of two unconditioned stimuli (UCS); 1) intra-oral milk infusions or 2) stroking with a sable-hair brush. These UCS's produce a common general response of increased behavioral activity, but different specific behaviors. For each UCS, additional pups received backward pairings of the CS and UCS, random pairings of the CS and UCS, CS only, UCS only, or no stimuli. Four hours later, pups received a two-odor choice test to assess the development of an odor preference and a CS-only test to assess the acquisition of conditioned responding (CR). The results of the two-odor choice test indicated that for both UCS's only forward pairings of the CS and UCS resulted in an odor preference. Similarly, the CS-only test showed that only forward pairings of the CS and either UCS were effective in producing CR's; pups that received forward pairings exhibited increased behavioral activity during presentations of the CS, which is an unconditioned response (UCR) to both UCS's. Only the forward paired CS-milk UCS group exhibited increased mouthing and probing during the CS only test; these are UCR's that occur to milk infusions but infrequently to the stroking UCS. These results demonstrate the development of similar conditioned odor preferences using behaviorally activating UCS's, but CR's which are specific to the form of the UCR.

Food-deprived infant rats respond to intra-oral infusions of milk with specific ingestive responses such as mouthing and probing as well as with more generalized behavioral activation (Hall, 1979a,b). This behavioral activation is characterized by vigorous paw treading, rolling, and locomotion and is not directed to the environmental stimuli eliciting the activation (Hall \& Bryan, 1980). Such undifferentiated activation is only characteristic of young pups and is not observed beyond the first week of life (Hall, 1979a,b).

Reprint requests should be sent to Regina M. Sullivan, Department of Psychobiology, University of California, Irvine, CA 92717.

Received for publication 15 September 1986

Revised for publication 16 January 1987

Accepted at Wiley 30 November 1987 
Behavioral activation may be related to the reward value of milk in early appetitive learning. For deprived pups, a classical conditioning procedure that pairs a novel odor (CS) with intra-oral infusions of milk is effective in producing both a conditioned odor preference (Johanson \& Hall, 1982; Johanson \& Teicher, 1980) and conditioned responses (CR's) such as mouthing, probing, and behavioral activation (Johanson, Polefrone, \& Hall, 1984). However, under conditions in which milk does not elicit behavioral activation, such as in nondeprived, dehydrated, or cold pups, learning is less apparent (Johanson et al., 1984). Thus, there exists a close relationship between behavioral activation and the occurrence of conditioning when milk is used as a reinforcer. This relationship has led to the conceptualization of behavioral activation as an external representation of positive affect in infants (Hall, 1982) and suggests that activation produced in other manners might be equivalently reinforcing.

Behavioral activation may be induced in infant rats by other forms of external stimulation, varying from very natural to unnatural. These include: stroking with a soft brush, tail pinching, introducing the odor of maternal saliva, milk odor, or brain stimulation (Moran, Lew, \& Blass, 1981; Moran, Schwartz, \& Blass, 1983; Pedersen \& Blass, 1982; Pedersen, Williams, \& Blass, 1982; Sullivan, Hofer, \& Brake, 1986a; Sullivan, Brake, Hofer, \& Williams, 1986b; Terry, \& Johanson, 1987; Wilson, Sullivan, \& Leon, 1985). For many of these other stimuli it has also been shown that pairing an odor with a stimulus that produces behavioral activation results in a learned preference for the odor (Sullivan \& Leon, 1986; Sullivan et al., 1986a), as well as enhancement of specific behaviors (Pedersen \& Blass, 1982; Pedersen et al., 1982; Sullivan et al., 1986b; Terry \& Johanson, 1987; Wilson, Sullivan, \& Leon, 1987).

Although these studies suggest that eliciting behavioral activation alone may be sufficient for reward functioning, conditioning has not been adequately examined with these activating procedures. For example, the above studies with stimulation-induced behavioral activation have generally used a continuous, simultaneous exposure to the odor and stimulation, preventing analysis of the relationship of activation to conditioning stimuli that is achieved by repeated temporal pairings in classical conditioning procedures (e.g., forward, backward, and randomized presentation of the odor and stimulation; Rescorla, 1967). The purpose of the present study was to assess the role of induced behavioral activation in the learning of classically conditioned responses, specifically comparing milk-elicited activation to tactile stimulation-induced activation.

Specifically, this experiment examined the relative abilities of milk and stroking to function as a reward in the acquisition of a conditioned odor preference and conditioned responding. Pups were trained in a classical conditioning procedure with the CS and one of the two UCS's. Later, they were tested on a two-odor choice test and a CS-only odor test.

\section{Methods}

The subjects were 120,6-day-old male and female pups from 12 litters born of Charles River CD rats mated in our colony. Following breeding, females were individually housed in plastic tubs $(48 \times 245 \times 20 \mathrm{~cm})$ containing pine chip bedding. Water and Purina Lab Chow were continuously available. The colony room was temperature $\left(21-23^{\circ} \mathrm{C}\right)$, and humidity controlled and on a $14: 10$ 
light : dark cycle. Females were checked for births at $1700 \mathrm{hrs}$ and litters found at that time were considered 0 days old. At 2 days of age, litters were culled to 10 pups with an even sex distribution. Only one pup from each litter was used in each experimental condition, with equal number of males and females in each condition.

An additional 16 male and female rats from 4 different litters were used to assess the unconditioned responses (UCR) to; 1) intra-oral infusions of milk, 2) stroking all areas of the body with a sable-hair brush, 3) presentation of cedar odor, and 4) no stimulus. These pups were observed for $1 \mathrm{hr}$, every $3 \mathrm{~min}$ for the occurrence of behavioral activation, mouthing, and probing; an observation schedule which coincided with the presentation of the stimuli. Details of the observation procedure are described under the conditioned stimulus (CS) only test.

\section{Conditioning Procedure}

Training consisted of 20 trials of CS/UCS presentations with an intertrial interval (ITI) of $3 \mathrm{~min}$, resulting in a training session lasting $1 \mathrm{hr}$. The presentation of the CS lasted $10 \mathrm{sec}$. An incubator fan operated continuously to clear the air of odor. The UCS lasted $5 \mathrm{sec}$. There were 10 training conditions; 1$) \mathrm{CS}-\mathrm{M}-$ forward pairings of cedar and milk; $10 \mathrm{sec}$ of odor with the last $5 \mathrm{sec}$ overlapping with a 5-sec milk infusion, 2) M-CS-backward presentations of milk and cedar; 5-sec milk infusions were followed by $10 \mathrm{sec}$ of cedar, 3) R-M-CS_randomized presentations of cedar and milk; the odor was presented every 3 min and the milk was presented on a variable interval schedule of $3 \mathrm{~min}, 4) \mathrm{M}$ - presentation of the 5 -sec milk infusion every $3 \mathrm{~min}, 5$ ) $\mathrm{CS}$ - presentation of cedar every $3 \mathrm{~min}, 6) \mathrm{N}$-naive, no presentations of cedar, milk, or stroking, 7) ST-presentation of $5 \mathrm{sec}$ of stroking every $3 \mathrm{~min}, 8$ ) R-ST-CS—randomized presentation of stroking and cedar; cedar was presented every $3 \mathrm{~min}$ and the stroking was presented on a variable interval schedule of $3 \mathrm{~min}$, 9) ST-CS_backward presentation of the cedar and stroking; $5 \mathrm{sec}$ of stroking was followed by $10 \mathrm{sec}$ of cedar, 10) CS-ST-forward pairings of cedar and stroking; $10 \mathrm{sec}$ of cedar with the last $5 \mathrm{sec}$ overlapping with $5 \mathrm{sec}$ of stroking. Following training, pups were removed from the training incubator, placed in individual styrofoam cups and placed in another warm $\left(33^{\circ} \mathrm{C}\right)$, moist incubator until testing.

Pups were deprived of maternal food and care for $18 \mathrm{hr}$ prior to treatment and housed in a warm $\left(32^{\circ} \mathrm{C}\right)$, moist incubator. Immediately prior to treatment, pups' bladders were voided and defecation stimulated by gently stroking the anogenital region with a soft brush (a procedure normally done by the mother). Treatment took place in individual transparent plastic containers $(12 \mathrm{~cm}$ diameter), within a warm $\left(32^{\circ} \mathrm{C}\right)$, moist incubator. To present the novel cedar odor (CS), a light air stream was passed through a vial containing $2 \mathrm{cc}$ of cedar wood oil (Fisher Scientific, Fair Lawn, N.J.) and directed through plastic tubing to exit $3 \mathrm{~cm}$ above the pup's head. Following odor delivery, a continuously operating fan cleared the odor from the incubator. Intra-oral infusions of milk (commercially available 'Half \& Half') were made through oral cannulas attached to delivery tubes from syringes driven by an infusion pump (Harvard Model 945). Stroking consisted of vigorous stroking of all surface areas of the pup's body with a soft sable-hair brush. The timing (described below) of both the odor and milk delivery was automatically controlled by a programmable timer (Chrontrol, Model 3A-1-2, Lindburg Enter- 
prises, San Diego, CA). The same timer signalled the experimenter for starting and stopping stroking stimulation.

\section{Testing}

Testing began 3 to $4 \mathrm{hr}$ after training. All pups received two tests; a two-odor choice test and a CS-only test. One half of the pups were given the two-odor choice test first; the other half were given the CS-only test first. No order effects were found for testing.

\section{Two-Odor Choice Test}

The test apparatus consisted of a $35 \times 20 \times 10 \mathrm{~cm}$ aluminum box with a wire mesh floor $(0.4 \times 0.4 \mathrm{~cm}$ grid $)$ divided in half by a $4-\mathrm{cm}$ neutral zone which ran the long length of the test box. Beneath the wire mesh floor, two containers of shavings were placed on either side of the neutral zone; one contained cedar-scented shavings ( $2 \mathrm{cc}$ of cedar oil : $200 \mathrm{cc}$ of wood shavings), the other contained an equal amount of unscented wood shavings. Pups received 5 consecutive 1-min trials in this apparatus. A trial began by placing the pup on the neutral zone, parallel to the long side of the test box. The amount of time the pup spent over each odor container was timed. Pups were considered over the odor when the two front paws and snout left the neutral zone. Between each trial, the pup was taken out of the apparatus and the floor was wiped clean with water. The direction in which the pup was placed back into the apparatus was counterbalanced between trails. At the end of the test, the total number of seconds over each odor was recorded.

\section{CS-only Test}

Pups were placed in individual, transparent containers (12 $\mathrm{cm}$ diameter) in a warm $\left(32^{\circ} \mathrm{C}\right)$, moist incubator. During the CS-only test, pups were given five 10-sec odor presentations (as described under "Training") with an ITI of 2 min. During the $10 \mathrm{sec}$ pre-CS and the $10 \mathrm{sec}$ CS, it was noted whether pups were mouthing (movement of the mouth and jaw for at least $2 \mathrm{sec}$ ) or probing (repetitive, forward, and lateral head movements in contact with and directed into the floor of the test container). The level of behavioral activity was also rated; 0 - not active; 1-movement of part of the body, e.g., face washing, head raising; 2movement of the entire body, e.g., head and limb movements, locomotion; 3extreme behavioral activity, e.g., rolling over, wall climbing (Hall, 1979b).

\section{Results}

\section{UCR's}

Both stroking and intra-oral milk infusions produced behavioral activation in infant rats in terms of increased activity (Figure 1a; $F=162, p<0.01$; post-hoc Tukey tests revealed the means of these groups were different from the mean of the odor and no stimulus group, $p<0.05$ ). Odor presentation produced little activity; values were similar to pups receiving no stimulus. High levels of mouthing (Fig. 1b, $F=32.65, p<0.01$ ) were only exhibited by pups receiving milk. Both milk and stroking elicited probing but significantly more probing 


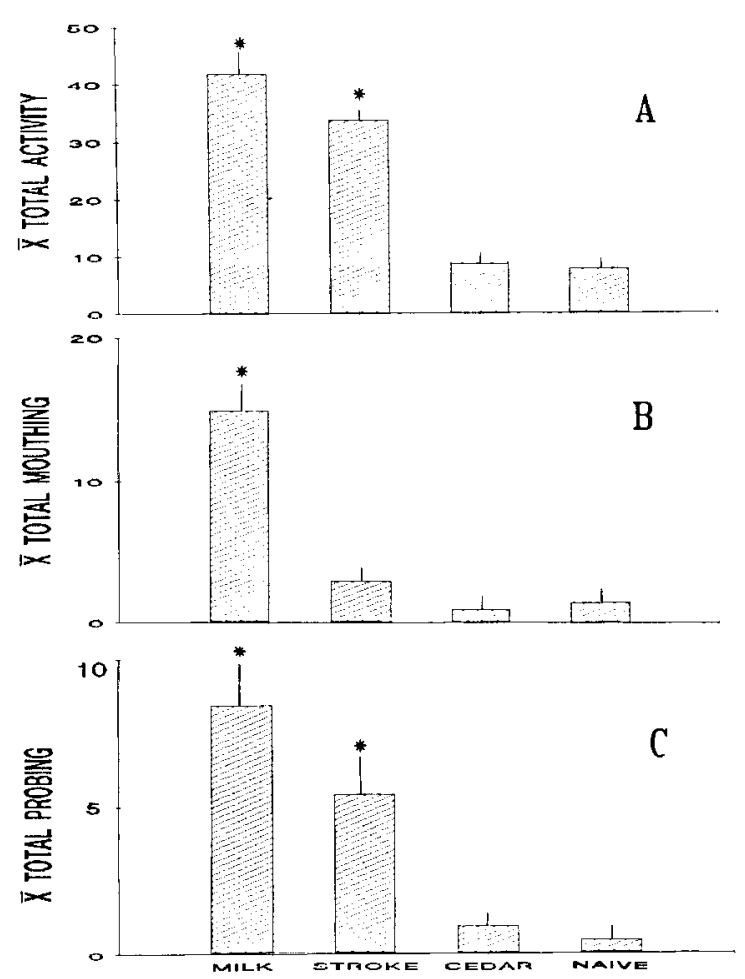

Fig. 1. Mean (+/-SE) total unconditioned responses of; a) behavioral activation, b) mouthing, and c) probing observed in response to intra-oral milk infusions, stroking the pups' body with a sable-hair brush, presentation of cedar odor, and no stimulus presentation. An asterisk indicates a significance level of at least $p<0.05$.

occurred in pups receiving milk than those being stroked (Fig. 1c, $F=14.09, p<$ 0.01). Post-hoc Tukey test revealed that the means of the milk and stroked groups each differed significantly from the mean of the cedar group; the means of the milk and stroked group differed significantly from one another $(p<0.05)$. Thus, behavioral activation is elicited by both intra-oral infusions of milk and stroking but the specific form of the activation differed. Milk reliably elicits mouthing, a behavior rarely exhibited in response to stroking. Probing was more frequent in response to milk infusions, though it was also elicited by stroking.

\section{Two-Odor Choice Test}

Forward pairings of the CS and either UCS were effective in producing an odor preference; pups in the CS-M and CS-ST training groups spent more time over the cedar odor than all other training groups (Fig. $2 ; F=8.156, p<0.01$ ). Post-hoc Tukey tests revealed that the mean of the CS-M and CS-ST groups each differed from all other treatment groups $(p<0.05)$. The means of CS-M and CS-ST did not differ from one another. There was no indication that odor experience in any relation to one of the activating UCS's, other than the forward conditioning, reduced the aversiveness of the odor CS or induced a preference to the odor CS. 


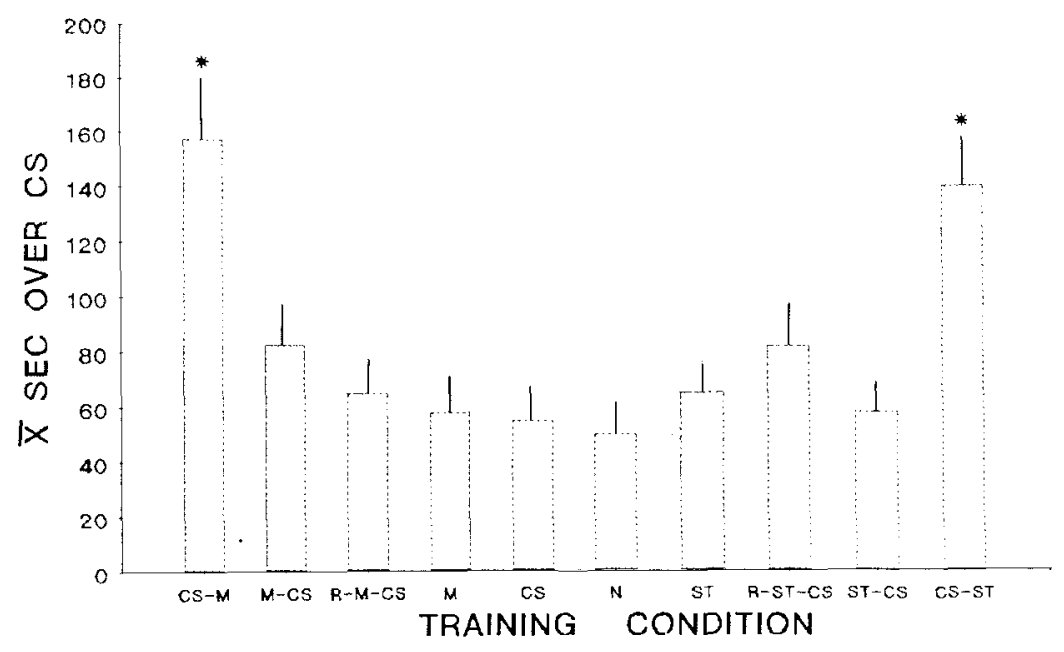

Fig. 2. Mean (+/-SE) number of seconds spent over the cedar CS in the two-odor choice test for the different training conditions; 1) CS-M-forward pairings of cedar and milk, 2) M-CS-backward pairings of milk and cedar, 3) R-M-CS_random pairings of milk and cedar, 4) CS_cedar only, 5) $\mathrm{M}$-milk only, 6) N-neither stimuli, 7) ST-stroking only, 8) R-ST-CS-random pairings of stroking and cedar, 9) ST-CS-backward pairings of stroking and cedar, and 10) CS-ST_forward pairings of cedar and stroking. An asterisk indicates a significance level of at least $p<0.05$ ).

\section{CS-Only Test}

Forward pairings of the CS and either UCS were effective in conditioning CR's to the presentation of odor. Pups in the CS-M and CS-ST training groups exhibited an increase in behavioral activation during presentation of the odor as compared to that exhibited just prior to the onset of the CS (Fig. 3a; $F=4.41 ; p<$ $0.01)$. Post-hoc Tukey tests revealed that the mean of the CS-M and CS-ST training groups each differed from all other treatment groups $(p<0.05)$. The mean of CS-M and CS-ST did not differ from one another. Additional CR's were conditioned in the CS-M training group; these pups exhibited an increase in both mouthing (Fig. 3b; $F=3.05 ; p<0.05$ ) and probing (Fig. 3c; $F=3.37 ; p<$ 0.05 ) during presentation of the odor as compared to that exhibited just prior to the onset of the CS. Neither of these responses was conditioned in the forward cedar and stroking group, despite the fact that probing was part of the UCR to stroking. The fact that significantly lower levels of probing were elicited by stroking than by milk infusions may be responsible for the inability to elicit probing as a CR following CS-ST training.

\section{General Discussion}

These results indicate that stroking, like oral infusions of milk, is an effective reinforcer in a classical conditioning paradigm utilizing explicit pairings and standard controls. Indeed, in terms of supporting both a conditioned odor preference and conditioned activation, stroking appeared very similar to milk in its reinforcing characteristics. Notably, only forward pairings of odor and stroking or milk resulted in conditioning, thus demonstrating stroking's function as a UCS (Pavlov, 1927; Rescorla, 1967).

Although previous studies have assessed the reinforcing effects of stroking in 

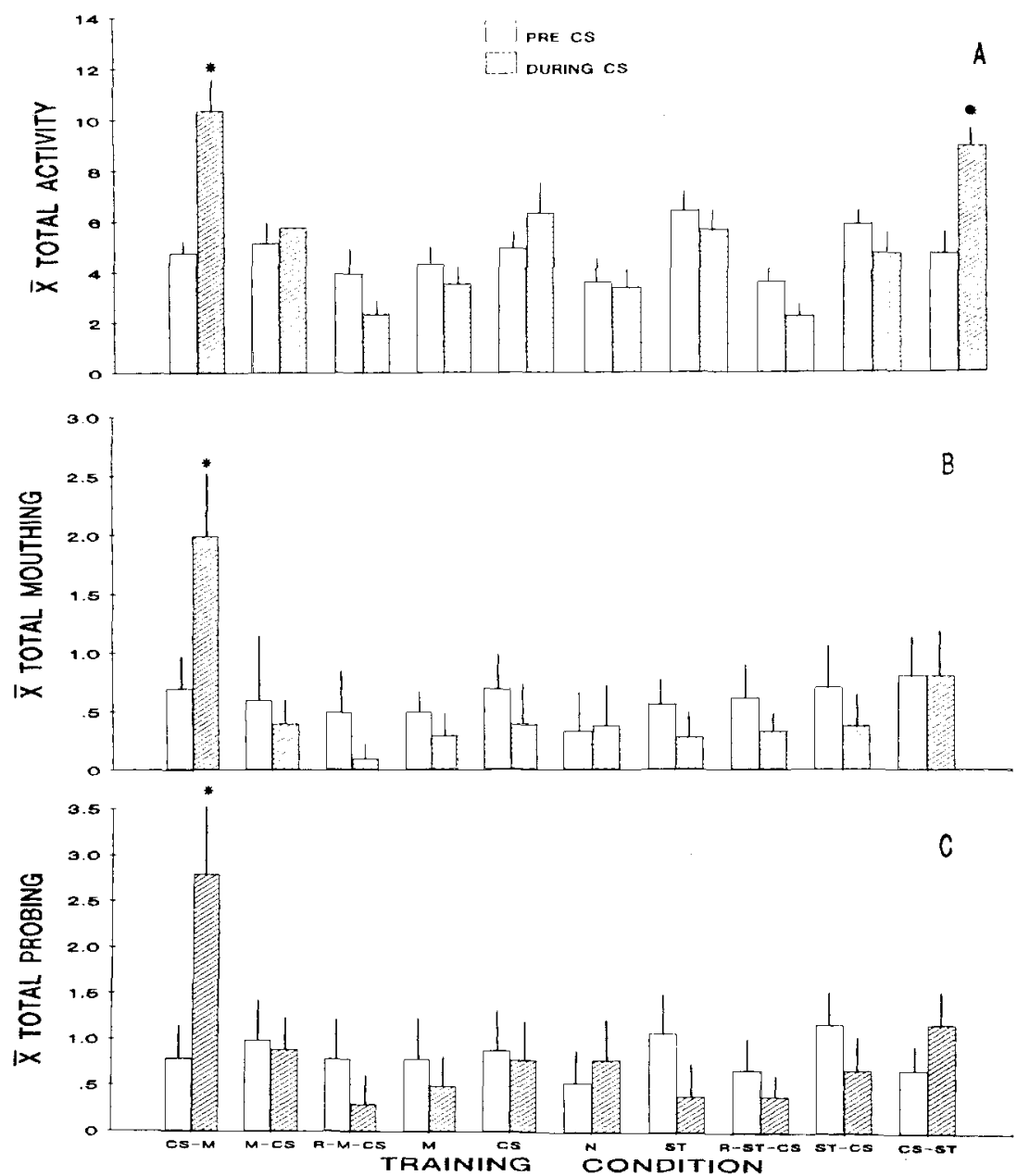

Fig. 3. Mean ( $+/-\mathrm{SE}$ ) total number of conditioned respondings of a) behavioral activity, b) mouthing, and c) probing before the odor (open bars) and during the odor (closed bars) during the CS-only test for each of the different training conditions (see Figure legend 2). An asterisk indicates a signficance level of at least $p<0.05$.

a classical conditioning paradigm, only one continuous presentation of the odor and stroking was used (Pedersen \& Blass, 1982; Pedersen et al., 1982; Sullivan et al., 1986a,b). Thus, only limited testing of stroking as a reward was possible. The present study replicates and extends these studies by further assessing the reinforcing role of stroking in two ways. First, the present study used repeated, discrete pairings of the odor and reward. This enabled the testing of two control groups which would not be expected to learn in classical conditioning; backward and randomized presentation of the odor and reinforcement (Rescorla, 1967). Moreover, pups in these control groups would be expected to perform at levels significantly different from naive and odor only pups if habituation or attentional mechanisms were responsible for the enhanced behaviors of pups in the forward odor and reinforcement condition. Since pups in the backward and randomized groups performance did not differ from the other learning control groups, the results of this paper indicate that stroking is indeed functioning as a reinforcer. Second, the present study compared the reinforcing value of stroking to a well 
documented, traditional reward-milk. The results indicates that stroking, when used as a reinforcer, produces learning as robust as that which results when milk is used as a reinforcer. Thus, the results of the present paper clearly indicate that stroking functions as a reward in a classical conditioning paradigm similar to that used with adults.

There are at least two possible reasons for the ability of stroking to function as a reward. First, it is possible that stroking, similarly to food, possesses specific biological relevance to pups. For instance, stroking is capable of altering specific biological processes (Butler, Suskind, \& Schanberg, 1968; Evoniuk, Kuhn, \& Schanberg, 1979; Kuhn, Butler, \& Schanberg, 1978). Second, and perhaps more likely, is that stroking's reinforcing properties may be nonspecific stimulation. Within the first week of life, a variety of stimuli (amphetamine injection, Pedersen et al., 1982; milk, Johanson, \& Hall, 1982, Johanson \& Teicher, 1980; milk odor, Terry and Johanson,1987; stroking, Pedersen \& Blass, 1982; Pedersen et al., 1982, Sullivan \& Leon, 1986, 1987; Sullivan et al., 1986a,b, Wilson, Sullivan \& Leon, 1987; electrical brain stimulation, Moran et al., 1981; the odor of maternal saliva and tailpinch, Sullivan et al., 1986b) appear to possess reinforcing value; that is, they are capable of producing an odor preference and/or specifically enhancing behavior. A common component in pups' responses to reinforcing stimuli is behavioral activation. Therefore, activation appears to be an important variable in the chain of responses elicited by reinforcing stimuli.

Behavioral activation itself, however, may not be reinforcing. Rather, a stimulus with reinforcing properties may also produce behavioral activation. Indeed, behavioral activation may simply be a window uniquely suited for viewing the arousal state of the infant and corresponds with a behavioral state which is conducive to learning. Thus, pups may be revealing the occurrence of affect or reward in their behavioral activation (Hall, 1982). Affect may be externalized in infant pups because the immature central nervous system lacks certain "inhibiting" or "directing" mechanisms. Indeed, the notion that behavioral activation may be an external representation of reward has support from neurophysiology. Electrical stimulation of the medial forebrain bundle (MFB), a traditional reward pathway, produces behavioral activation in infant rats (Moran et al., 1981; Moran et al., 1983). This stimulation is also capable of functioning as a reward in an operant conditioning study in infant rats (Moran et al., 1981).

The results of the present paper, along with the results of other laboratories, suggests that numerous "activating" stimuli, many normally received by pups from the mother, have reinforcing value. In particular, a variety of maternal behaviors, such as licking or stepping on pups, appear likely to be capable of forming pups' attraction to odors. Thus, pups experiencing repeated pairings of maternal odors and maternal behaviors consequently develop an attraction to those maternal odors (Alberts \& May, 1984; Galef \& Kaner, 1980; Leon, Galef, \& Behse, 1972). This method of olfactory preference development would enable a wide variety of stimuli to function as a UCS and would ensure attraction to the pups' source of food and warmth, its mother.

\section{Notes}

This research was supported by NICHD Grant HD 17458 to W.G.H. and NICHD Grant HD 06818 to R.M.S. Present address for R.M.S. is University of California, Department of Psychobiology, Irvine CA 92717. The authors would like to thank D. A. Wilson for his comments on an earlier draft of this paper. 


\section{References}

Alberts, J. R., and May, B. (1984). Nonnutritive, thermotactile induction of filial huddling in rat pups. Dev. Psychobiol., 17: 161-181.

Butler, S. R., Suskind, M. R., and Schanberg, S. M. (1968). Maternal behavior as a regulator of polyamine biosynthesis in brain and heart of the developing rat pup. Science, 199: 445-447.

Evoniuk, G. E., Kuhn, C. M., and Schanberg, S. M. (1979). The effect of tactile stimulation on serum growth hormone and tissue ornithine decarboxylase in rat pups. Comm. Psychopharmacol., 3: 363-370.

Galef, B. G., and Kaner, H. C. (1980). Establishment and maintenance of preference for natural and artificial olfactory stimuli in juvenile rats. J. Comp. Physiol. Psychol., 94: 588-595.

Hall, W. G., (1979a). Feeding and behavioral activation in infant rats. Science, 205: 206-209.

Hall, W. G., (1979b). The ontogeny of feeding in rats. I. Ingestive and behavioral responses to oral infusions. J. Comp. Physiol. Psychol., 93: 977-1000.

Hall, W. G. (1982). Feeding, food reward, and learning: An appetitive system for ingestive behavior in infant rats. In: Symposium Neuroontogeneticium, (S. Trojan and J. Mourek, Eds.), Charles University Press: Prague.

Hall, W. G., and Bryan, E. (1980). The ontogeny of feeding in rats: Il. Independent ingestive behavior. J. Comp. Physiol. Psychol., 94: 746-756.

Johanson, I. B., and Hall, W. G. (1982). Appetitive conditioning in neonatal rats: Conditioned orientation to an odor. Dev. Psychobiol., 15: 379-397.

Johanson, I. B., Polefrone, J. M., and Hall, W. G. (1984). Appetitive conditioning in neonatal rats: Conditioned ingestive responding to stimuli paired with oral infusions of milk. Dev. Psychobiol. 17: 357-381.

Johanson, I. B., and Teicher, M. (1980). Classical conditioning of an odor preference in 3-day-old rats. Behav. Neur. Biol., 29: 132-136.

Kuhn, C. M., Butler, S. R., and Schanberg, S. M. (1978). Selective depression of serum growth hormone during maternal deprivation in rat pups. Science, 201: 1034-1036.

Leon, M., Galef, B. G., and Behse, J. H. (1972). Establishment of pheromonal bonds on diet choice in young rats by odor preexposure. Physiol. Behav., 18: 387-391.

Moran, T. H., Lew, M. F., and Blass, E. M. (1981). Intracranial self-stimulation in 3-day-old rat pups. Science, 214: 1366-1368.

Moran, T. H., Schwartz, G. J., and Blass, E. M. (1983). Stimulation-induced ingestion in neonatal rats. Dev. Brain Res., 7: 197-204.

Pavlov, I. P. (1927). Conditioned reflexes. Oxford University Press: New York.

Pedersen, P. E., and Blass, E. M. (1982). Prenatal and postnatal determinants of the first suckling episode in albino rats. Dev. Psychobiol., 15: 349-355.

Pedersen, P. E., Williams, C. L., and Blass, E. M. (1982). Activation and odor conditioning of suckling behavior in 3-day-old albino rats. J. Exp. Psych.: Anim. Beh. Proc., 8: 829-841.

Rescorla, R. A. (1967). Pavlovian conditioning and its proper control procedures. Psychol. Rev., 74: $71-80$.

Sullivan, R. M., Brake, S. C., Hofer, M. A., and Williams, C. L. (1986b). Huddling and independent feeding in neonatal rats can be facilitated by a conditioned change in behavioral state. Dev. Psychobiol., 19: 625-635.

Sullivan, R. M., Hofer, M. A., and Brake, S. C. (1986a). Olfactory-guided orientation in neonatal rats is enhanced by a conditioned change in behavioral state. Dev. Psychobiol., 19: 615-623.

Sullivan, R. M., and Leon, M. (1986). Early olfactory learning induces an enhanced olfactory bulb response in young rats. Dev. Brain Res., 27: 278-282.

Sullivan, R. M., Wilson, D. A. and Leon, M. (1987). One-trial olfactory learning enhances olfactory bulb responses to an appetitive conditioned odor in 7-day-old rats. Devel. Brain Res., 35:307311.

Terry, L. M., and Johanson, I. B. (1987). Olfactory influences on the ingestive behavior of infant rats. Devel. Psychobiol., 20:313-332.

Wilson, D. A., Sullivan, R. M., and Leon, M. (1985). Odor familiarity alters mitral cell response in the olfactory bulb of neonatal rats. Dev. Brain Res., 22: 314-317.

Wilson, D. A., Sullivan, R. M., and Leon, M. (1987). Single unit analysis of postnatal olfactory learning: Modified olfactory bulb output response patterns to learned attractive odors. $J$. Neurosci., 7: 3154-3162. 